EESTI NSV TEADUSTE AKADEEMIA TOIMETISED. 25. KOIDE FUOSIKA * MATEMAATIKA, 1976, NR. 3

ИЗВЕСТИЯ АКАДЕМИИ НАУК ЭСТОНСКОП ССР. ТОМ 25 ФИЗИҚА * МАТЕМАТИКА. 1976, № 3

\title{
ЭХО-СИГНАЛ КОНЕЧНОГО ИМПУЛЬСА ОТ ЗАПОЛНЕННОЙ ЖИДКОСТЬЮ ЦИЛИНДРИЧЕСКОЙ ОБОЛОЧКИ
}

\begin{abstract}
Предлагается способ вычисления эхо-сигнала конечного плоского импульса давления от заполненной жидкостью упругой цилиндрической оболочки, находящейся в безгранично жидкой среде. Как среда, окружающая оболочку, так и жидкость, заполняющая ее, моделируются по линейной теории идеальной сжимаемой жидкости. Движение оболочки описывается с помощью линейной теории тонких оболочек типа Тимошенко. Задача решается путем применения интегрального преобразования Фурье по времени и преобразования Ватсона по угловой координате. Приводятся механизмы образования различных составляющих эхо-сигнала.
\end{abstract}

Первые работы по дифракщии акустических волн на цилиндрических оболочках появились в $50-x$ годах $[1,2]$. В этих работах решение отыскивалось в рядах по собственным функциям. В последнее время наряду с разложением по собственным функциям стал развиваться метод разложения решения по модам $\left[{ }^{3-9}\right]$. В работе $\left[{ }^{6}\right]$ рассмотрено вычисление отраженной составляющей эхо-сигнала, а также его составляющих, излученных ползущими и периферическими волнами, от пустых цилиндрических оболочек. Вклады в эхо-сигнал волн, проходящих как жидкий, так и упругий цилиндр, изучены методом преобразования Ватсона в $\left[{ }^{7,8}\right]$. В работе $\left[{ }^{9}\right]$ исследовано влияние толщины стенки и наличия жидкости на отдельные моды колебания цилиндрической оболочки.

В настоящей работе исследуется эхо-сигнал от цилиндрической оболочки, заполненной жидкостью, причем, кроме указанных выше составляющих эхо-сигнала, учитываются вклады волн, распространяющихся частично в оболочке как периферические и частично как проходящие жидкий заполнитель.

1. Постановка за д а ч и. Введем обозначения: $c$, o - скорость звука и плотность среды, окружающей оболочку; $c_{0}, 0_{0}-$ скорость звука и плотность заполнителя; $\tau$ - безразмерное время $(\tau=c t / R$, $t$ - время, $R$ - радиус оболочки) $; r, \vartheta$ - безразмерные (все размеры длины выражены в радиусах $R$ срединной поверхностй оболочки) цилиндрические координаты, ось которых совпадает с осью оболочки $(\vartheta=0$ для радиус-вектора, направленного перпендикулярно к плоскости отсчета, т. е. к плоскости, где находится фронт зондирующего импульса, когда $t=0) ; p_{i}(r, \vartheta, \tau)-$ зондирующий импульс давления; $p_{e}(r, \vartheta, \tau)$ - эхо-сигнал (рассеянное давление); $p_{0}(r, \vartheta, \tau)$ - давление в заполнителе; $h$ - толщина оболочки, $E, v, \varrho_{1}$ - модуль упругости, коэффициент Пуассона и плотность материала оболочки; $u, \psi, w-$ перемещения, используемые в теории оболочек типа Тимошенко; $q$ - нормальное давление, действующее на поверхность оболочки; $r_{0}$ - без- 
размерное расстояние от плоскости отсчета до оси оболочки; $A_{0}, f-$ постоянная, определяющая амплитуду, и закон изменения давления в зондирующем импульсе; $l$ - безразмерное расстояние от плоскости отсчета; $\tau_{p}$ - безразмерная продолжительность зондирующего импульса; $H$ - единичная функция Хевисайда.

Пусть в безграничной идеальной сжимаемой среде распространяется плоский зондирующий импульс давления

$$
\begin{aligned}
& p_{i}=A_{0} f(\tau-l)\left[H(\tau-l)-H\left(\tau-l-\tau_{p}\right)\right], \\
& l=r_{0}-r \cos \vartheta .
\end{aligned}
$$

Импульс (1.1), падая на оболочку, не только отражается от ее поверхности, но и генерирует в оболочке и заполнителе упругие волны, которые, в свою очередь, возбуждают в окружающей среде различные составляющие эхо-сигнала.

Для описания упругих волн в оболочке используются уравнения теории оболочек типа Тимошенко $\left[{ }^{10}\right]$ :

$$
\sum_{j=1}^{3} L_{i j} \Phi_{j}=-\delta_{i 3} \alpha^{-1} c^{-2} Q^{-1} q, \quad i=1,2,3,
$$

$$
\begin{aligned}
& L_{11}=\left(1+a^{2}\right)\left(\frac{\partial^{2}}{\partial \vartheta^{2}}-k_{v}^{2} x^{2}\right)-\beta^{2} \frac{\partial^{2}}{\partial \tau^{2}}, \quad L_{12}=a^{2}\left(-\frac{\partial^{2}}{\partial \vartheta^{2}}+k_{\vartheta}^{2} x^{2}-\right. \\
&\left.-\beta^{2} \frac{\partial^{2}}{\partial \tau^{2}}\right)+k_{\vartheta}^{2} x^{2}, \quad L_{13}=\left(1+a^{2}\right)\left(1+k_{v}^{2} x^{2}\right) \frac{\partial}{\partial \vartheta}, \\
& L_{21}= a^{2}\left(-\frac{\partial^{2}}{\partial \vartheta^{2}}+k_{\vartheta}^{2} x^{2}-\beta^{2} \frac{\partial^{2}}{\partial \vartheta^{2}}\right)+k_{\vartheta}^{2} x^{2}, \quad L_{22}=a^{2}\left(\frac{\partial^{2}}{\partial \vartheta^{2}}-k_{\vartheta}^{2} x^{2}-\right. \\
&\left.-\beta^{2} \frac{\partial^{2}}{\partial \tau^{2}}\right)-k_{\vartheta}^{2} x^{2}, \quad L_{23}=-\left[\left(1+a^{2}\right) k_{v}^{2} x^{2}+a^{2}\right] \frac{\partial}{\partial \vartheta}, \\
& L_{31}=-\left(1+a^{2}\right)\left(1+k_{\vartheta}^{2} x^{2}\right) \frac{\partial}{\partial \vartheta}, \quad L_{32}=\left[\left(1+a^{2}\right) k_{v}^{2} x^{2}+a^{2}\right] \frac{\partial}{\partial \vartheta}, \\
& L_{33}=\left(1+a^{2}\right)\left(k_{\vartheta}^{2} x^{2} \frac{\partial^{2}}{\partial \vartheta^{2}}-1\right)-\beta^{2} \frac{\partial^{2}}{\partial \tau^{2}}, \quad k_{\vartheta}^{2}=\frac{\pi^{2}}{12}, \quad a^{2}=\frac{h^{2}}{12 R^{2}}, \\
& x^{2}= \frac{1-v}{2}, \quad \beta^{2}=\frac{(1-v) \varrho_{1} c^{2}}{E}, \quad \alpha=\frac{h_{\varrho 1}}{R \varrho \beta^{2}}, \quad \Phi_{1}=\frac{u}{R}, \\
& \Phi_{2}=\psi, \quad \Phi_{3}=\frac{w}{R} .
\end{aligned}
$$

Эхо-сигнал $p_{e}$ и давление $p_{0}$ в заполнителе должны удовлетворять соответственно уравнениям

$$
\begin{gathered}
\nabla^{2} p_{e}-\frac{\partial^{2} p_{e}}{\partial \tau^{2}}=0, \quad \nabla^{2} p_{0}-\beta_{0}^{2} \frac{\partial^{2} p_{0}}{\partial \tau^{2}}=0 \\
\nabla^{2}=\frac{1}{r} \frac{\partial}{\partial r}\left(r \frac{\partial}{\partial r}\right)+\frac{1}{r^{2}} \frac{\partial^{2}}{\partial \vartheta^{2}}, \quad \beta_{0}=\frac{c}{c_{0}}
\end{gathered}
$$

Краевые условия по координате $r$ следующие: в бесконечности ус- 
ловие излучения

$$
\lim \sqrt{r}\left(\frac{\partial}{\partial r}-\frac{\partial}{\partial \tau}\right) p_{e}=0
$$

на срединной поверхности оболочки условия контакта

$$
\begin{aligned}
& {\left[\frac{\partial^{2} w_{i}}{\partial \tau^{2}}\right]_{r=1}+\left[\frac{\partial^{2} w_{e}}{\partial \tau^{2}}\right]_{r=1}-\frac{\partial^{2} w}{\partial \tau^{2}}=0,} \\
& {\left[\frac{\partial^{2} w_{0}}{\partial \tau^{2}}\right]_{r=1}-\frac{\partial^{2} w}{\partial \tau^{2}}=0}
\end{aligned}
$$

и на оси оболочки условие ограниченности решения.

Ускорения в жидкостях в (1.5) определяются соотношениями

$\frac{\partial^{2} w_{i}}{\partial \tau^{2}}=-\frac{R}{\varrho c^{2}} \frac{\partial p_{i}}{\partial r}, \quad \frac{\partial^{2} w_{e}}{\partial \tau^{2}}=-\frac{R}{\varrho c^{2}} \frac{\partial p_{e}}{\partial r}, \quad \frac{\partial^{2} w_{0}}{\partial \tau^{2}}=-\frac{R}{\varrho_{0} c^{2}} \frac{\partial p_{0}}{\partial r}$,

а давление $q$, действующее на поверхность оболочки в (1.2), вычисляется по формуле

$$
q=-\left[p_{i}+p_{e}-p_{0}\right]_{r=1} .
$$

Краевыми условиями по углу $\vartheta$ являются условия периодичности и условия ограниченности решения.

Начальные условия в обычном смысле не формулируются, но поскольку в качестве преобразования по времени используется преобразование Фурье, то при $\tau \rightarrow \pm \infty$ все компоненты решения предполагаются стремящимися к нулю.

2. Решения в пространстве изображения. Для решения уравнений (1.2) и (1.3) используется преобразование Фурье по времени в виде

$$
\begin{aligned}
& p(r, \vartheta, \tau)=\int_{-\infty}^{\infty} p^{F}(r, \vartheta ; \omega) \mathrm{e}^{-i \omega \tau} d \omega, \\
& p^{F}(r, \vartheta ; \omega)=\frac{1}{2 \pi} \int_{-\infty}^{\infty} p(r, \vartheta, \tau) \mathrm{e}^{i \omega \tau} d \tau .
\end{aligned}
$$

Зондирующий импульс (1.1) в пространстве преобразования Фурье в координатах $r, \vartheta$ может быть представлен в виде

$$
\begin{gathered}
p_{i}^{F}=A_{0} f^{F} \sum_{m=0}^{\infty} \varepsilon_{m} a_{m} J_{m}(\omega r) \cos (m \vartheta), \\
f^{F}=\frac{1}{2 \pi} \int_{0}^{\tau_{p}} f(\tau) \mathrm{e}^{i \omega \tau} d \tau, \quad a_{m}=\mathrm{e}^{-i m \pi / 2} \mathrm{e}^{i \omega r_{0}}, \quad \varepsilon_{m}=2-\delta_{m 0},
\end{gathered}
$$

где $J_{m}-$ цилиндрическая функция Бесселя первого рода.

Компоненты решения уравнений (1.2) и (1.3) в пространстве преобразования Фурье, удовлетворяющие условиям излучения (1.4), периодичности и ограниченности решения, могут быть найдены в виде рядов

$$
p_{e}^{F}=A_{0} f^{F} \sum_{m=0}^{\infty} \varepsilon_{m} a_{m} x_{m} H_{m}^{(1)}(\omega r) \cos (m \vartheta),
$$




$$
\begin{aligned}
& p_{0}^{F}=A_{0} f^{F} \sum_{m=0}^{\infty} \varepsilon_{m} a_{m} y_{m} J_{m}\left(\beta_{0} \omega r\right) \cos (m \vartheta) \\
& \Phi_{1}^{F}=\sum_{m=0}^{\infty} \psi_{1 m} \sin (m \vartheta) \\
& \Phi_{2}^{F}=\sum_{m=0}^{\infty} \psi_{2 m} \sin (m \vartheta) \\
& \Phi_{3}^{F}=\sum_{m=0}^{\infty} \psi_{3 m} \cos (m \vartheta)
\end{aligned}
$$

Подставляя (2.2) и (2.3) в преобразованные по формуле (2.1) уравнения (1.2) и условия контакта (1.5), получим для коэффициентов $x_{m}$ формулу

$$
\begin{gathered}
x_{m}=-\frac{\omega A_{33} J_{m}(\omega)-\left(\alpha D+\alpha_{0} D_{0}\right) \partial J_{m}(\omega) / \partial \omega}{\omega A_{33} H_{m}^{(1)}(\omega)-\left(\alpha D+\alpha_{0} D_{0}\right) \partial H_{m}^{(1)}(\omega) / \partial \omega}, \\
D=\operatorname{det}\left|a_{i j}\right|, \quad i, j=1,2,3
\end{gathered}
$$

$$
\begin{gathered}
a_{11}=\left(1+a^{2}\right)\left(-m^{2}-k_{v}^{2} x^{2}\right)+\beta^{2} \omega^{2}, \quad a_{12}=a^{2}\left(m^{2}+k_{v}^{2} x^{2}-\beta^{2} \omega^{2}\right)+k_{0}^{2} x^{2}, \\
a_{13}=-\left(1+a^{2}\right)\left(1+k_{v}^{2} x^{2}\right) m, \quad a_{21}=a^{2}\left(m^{2}+k_{0}^{2} x^{2}+\beta^{2} \omega^{2}\right)+k_{0}^{2} x^{2},
\end{gathered}
$$

$a_{22}=a^{2}\left(-m^{2}-k_{v}^{2} x^{2}+\beta^{2} \omega^{2}\right)-k_{v}^{2} x^{2}, \quad a_{31}=-\left(1+a^{2}\right)\left(1+k_{0}^{2} x^{2}\right) m$,

$a_{32}=\left[\left(1+a^{2}\right) k_{v}^{2} x^{2}+a^{2}\right] m, \quad a_{33}=\left(1+a^{2}\right)\left(-k_{v}^{2} x^{2} m^{2}-1\right)+\beta^{2} \omega^{2}$,

$D_{0}={ }_{\omega} A_{33} J_{m}\left(\beta_{0 \omega}\right)\left[\partial J_{m}\left(\beta_{0} \omega\right) / \partial\left(\beta_{0} \omega\right)\right]^{-1}, \quad \alpha_{0}=\varrho_{0} C_{0} \varrho^{-1} C^{-1}$.

Здесь $A_{33}$ - соответствующее алгебраическое дополнение определителя $D$.

Для представления эхо-сигнала в физически хорошо интерпретируемых рядах по периферическим и ползущим волнам используется преобразование Зоммерфельда-Ватсона в виде [ $\left.{ }^{6}\right]$

$$
\sum_{m=0}^{\infty} \varepsilon_{m} F(m, \omega) \cos m \vartheta=i \int_{\Gamma} F(\mu, \omega) \sin ^{-1} \mu \pi \cos [\mu(\pi-\vartheta)] d \mu,
$$

где контур интегрирования $\Gamma$ охватывает положительную часть вещественной оси по часовой стрелке на комплексной плоскости $z$.

Используя (2.5) и соотношения

$$
\begin{gathered}
J_{\mu}(x)=\frac{1}{2}\left[H_{\mu}^{(1)}(x)+H_{\mu}^{(2)}(x)\right], \\
\cos [\mu(\pi-\vartheta)]=\frac{1}{2}\left[\mathrm{e}^{i \mu(\pi-\vartheta)}+\mathrm{e}^{-i \mu(\pi-\vartheta)}\right], \\
\sin ^{-1} \mu \pi=-2 i \mathrm{e}^{i \mu \pi} \sum_{n=0}^{\infty} \mathrm{e}^{2 i \mu \pi n},
\end{gathered}
$$

можем представить изображение эхо-сигнала (2.3) в виде 


$$
p_{e}^{F}=-\frac{1}{2} A_{0} f^{F} \sum_{k=1}^{2} \sum_{n=0}^{\infty} \int_{\Gamma} a_{\mu}\left[1+W H_{\mu}^{(2)}(\omega) / H_{\mu}^{(1)}(\omega)\right] S d \mu
$$

где

$$
\begin{array}{cc}
S=\mathrm{e}^{i \mu \vartheta_{n k}} H_{\mu}^{(1)}(\omega r), & W=F_{0}^{-1} F_{1}-T U V F_{0}^{-2}, \\
U=\left(1-V F_{2} F_{0}^{-1}\right)^{-1}, & V=H_{\mu}^{(1)}\left(\beta_{0} \omega\right) / H_{\mu}^{(2)}\left(\beta_{0} \omega\right),
\end{array}
$$

$F_{0}=\left(\omega A_{33}-\alpha D E_{1}\right) G_{2}-\alpha_{0} \omega A_{33} E_{1}, \quad F_{1}=\left(\omega A_{33}-\alpha D E_{2}\right) G_{2}-\alpha_{0 \omega} A_{33} E_{2}$,

$F_{2}=-\left(\omega A_{33}-\alpha D E_{1}\right) G_{1}+\alpha_{0} \omega A_{33} E_{1}, \quad T=\alpha_{0} \omega^{2} A_{33}\left(E_{1}-E_{2}\right)\left(G_{1}-G_{2}\right)$,

$\left.E_{1,2}=\left[\partial \underset{\mu}{H_{(1,2)}^{(\omega)}(\omega) / \partial \omega}\right] / H_{\mu}^{(1,2)}(\omega), \quad G_{1,2}=\left[\partial \underset{\mu}{H_{(1,2)}}\left(\beta_{0} \omega\right) / \partial\left(\beta_{0} \omega\right)\right)\right] / H_{\mu}^{(1,2)}\left(\beta_{0} \omega\right)$,

$\vartheta_{n 1}=\vartheta+2 n \pi, \quad \vartheta_{n 2}=2 \pi-\vartheta+2 n \pi$.

Здесь $a_{\mu}, D, A_{33}$ вычисляются соответственно по формулам (2.2), (2.4) при замене $m$ на $\mu$.

Отношения $F_{1} F_{0}^{-1}, F_{2} F_{0}^{-1}, T F_{0}^{-2}$ в $(2.8)$ имеют вполне определенный смысл: из них первые два - это коэффициенты внешнего и внутреннего отражения от поверхности оболочки, а третье - произведение коэффициентов прохождения из окружающей среды в заполнитель и обратно, из заполнителя в окружающую среду. Следует обратить внимание на то, что при каждом прохождении через оболочку часть энергии проходящих волн расходуется на возбуждение волн, распространяющихся в оболочке, поэтому связь между соответствующими коэффициентами прохождения и отражения является более сложной, чем в случае границы раздела двух сред.

Если опустить единицу в квадратных скобках (2.7) и представить функцию $U$ в виде ряда

$$
U=\sum_{j=1}^{\infty}\left(V F_{2} F_{0}^{-1}\right)^{j-1}
$$

изображение эхо-сигнала (2.7) примет вид

$$
\begin{gathered}
p_{e}^{F}=\frac{1}{2} A_{0} f^{F} \sum_{k=1}^{2} \sum_{n=0}^{\infty} \int_{\Gamma}\left(\frac{F_{1}}{F_{0}}-\sum_{j=1}^{\infty} T \frac{V^{j} F_{2}^{j-1}}{F_{0}^{j+1}}\right) K d \mu, \\
K=a_{\mu} H_{\mu}^{(1)-1}(\omega) H_{\mu}^{(2)}(\omega) H_{\mu}^{(1)}(\omega r) \mathrm{e}^{i \mu \theta_{n k} .}
\end{gathered}
$$

3. О бр а щени е пр еобр азованй й. Преобразование Зоммерфельда-Ватсона обращается приближенно методом перевала. Для этого быстроизменяющиеся функции $V$ и $K$ в $(2.10)$ заменяются их приближенными выражениями. Подставляя асимптотические представления

$$
\begin{aligned}
& H_{\mu}^{(1,2)}(\omega)=\left[\frac{2}{\pi \omega\left(1-z^{2}\right)^{1 / 2}}\right]^{1 / 2} \mathrm{e}^{ \pm i\left[\omega\left(\sqrt{1-z^{2}}-z \arccos z\right)-\pi / 4\right]}, \\
& z=\mu \omega^{-1}
\end{aligned}
$$

в формулы $(2.8)$ и $(2.10)$, имеем

$V=\exp \left\{2 i \omega \beta_{0}\left[\left(1-z^{2} \beta_{0}^{-2}\right)^{1 / 2}-z \beta_{0}^{-1} \arccos \left(z \beta_{0}^{-1}\right)\right]-i \pi / 2\right\}$,

$$
K=\left[\frac{2}{\pi \omega r}\right]^{1 / 2} N \exp \left\{i \omega\left[d_{0}+z\left(g_{0}+\vartheta_{n k}\right)\right]-i \pi / 4\right\}
$$




$$
\begin{aligned}
& N=\left(1-z^{2} r^{-2}\right)^{-1 / 4}, \quad d_{0}=\left(r^{2}-z^{2}\right)^{1 / 2}-r_{0}-2\left(1-z^{2}\right)^{1 / 2}, \\
& g_{0}=2 \arccos z-\arccos \left(z r^{-1}\right)-\pi / 2 .
\end{aligned}
$$

Подставляя (3.2) в $(2.10)$, получим изображение эхо-сигнала в виде

$$
p_{e}^{F}=A_{0} \frac{1}{\sqrt{2 \mathrm{r}}} f^{F} \sum_{k=1}^{2} \sum_{n=0}^{\infty} \sum_{j=0}^{\infty} T_{j n k},
$$

где $T_{\text {jnk }}$ при $j=0$ вычисляется по формуле

$$
T_{0 n k}=-\mathrm{e}^{i \alpha_{0}} \int_{\Gamma_{z}}\left(\frac{i \omega}{\pi}\right)^{1 / 2} N \frac{F_{1}}{F_{0}} \mathrm{e}^{i \omega \varphi_{n k}^{0}(z)} d z
$$

и при $j=1,2,3, \ldots$ - по формуле

$$
T_{j n k}=\mathrm{e}^{i \alpha \rho} \int_{\Gamma_{z}}\left(\frac{i \omega}{\pi}\right)^{1 / 2} N T \frac{F_{2}^{j-1}}{F_{0}^{j+1}} \mathrm{e}^{i \omega \varphi_{n k}^{\prime}(z)} d z .
$$

В формулах (3.4), (3.5) при любом значении $j$

$$
\begin{aligned}
& \varphi_{n k}^{j}(z)=d_{j}(z)+z\left[g_{j}(z)+\vartheta_{n k}\right], \quad \alpha_{j}=-j \pi / 2, \\
& d_{j}(z)=d_{0}+2 j \beta_{0}\left(1-z^{2} \beta_{0}^{-2}\right)^{1 / 2}, \\
& g_{j}(z)=g_{0}-2 j \arccos \left(z \beta_{0}^{-1}\right) .
\end{aligned}
$$

Контур интегрирования $\Gamma_{z}$ имеет такой же вид на плоскости $z$, что и контур $\Gamma$ на плоскости $\mu$.

Координаты $z_{\text {jnk }}$ седловых точек интегралов (3.4), (3.5) определяются из уравнения

$$
g_{j}(z)+\vartheta_{n k}=0
$$

причем при вычислении интеграла (3.4) следует брать $j=0$.

Уравнение (3.6) имеет решения $z=z_{j n k}$ лишь при выполнении следующих условий:

при $j=0$

$$
0 \leqslant \theta_{n k} \leqslant \arccos \left(r^{-1}\right)+\pi / 2,
$$

при $j=1,2, \ldots, \beta_{0} \leqslant 1$

$$
\arccos \left(\beta_{0} r^{-1}\right)+\pi / 2-2 \arccos \left(\beta_{0}\right) \leqslant \theta_{n k} \leqslant j \pi,
$$

при $j=1,2, \ldots, \beta_{0} \geqslant 1$

$$
\arccos \left(r^{-1}\right)+\pi / 2+2 j \arccos \left(\beta_{0}^{-1}\right) \leqslant \vartheta_{n k} \leqslant j \pi .
$$

Седловые точки интегралов (3.4) и (3.5) располагаются на вещественной оси $z$ между 0 и 1. Для вычисления интегралов (3.4) контур $\Gamma_{z}$ сначала заменяется контуром $\Gamma$, и поскольку во второй и четвертой четвертях нет особых точек, то $\Gamma$, в свою очередь, будет заменяться контуром, состоящим из участков $\Gamma_{\infty}, \Gamma_{0 n k}$ (рис. $\left.1, a\right)$. Для интегралов (3.5) контур $\Gamma_{z}$ заменяется контуром, состоящим из участков $\Gamma_{\infty}, \Gamma_{j n k}$ (рис. $1, \sigma)$. Вкладами участков $\Gamma_{\infty}, \Gamma_{\infty}^{\prime}$ контуров интегрирования можно пренебречь.

Необходимо отметить, что при вычислении интегралов (3.4) и (3.5) наряду с вкладами седловых точек следует учитывать и вклады полюсов первой четверти плоскости $z$, расположенных слева от седловой

4 ENSV TA Toimetised F* M-3 1976 

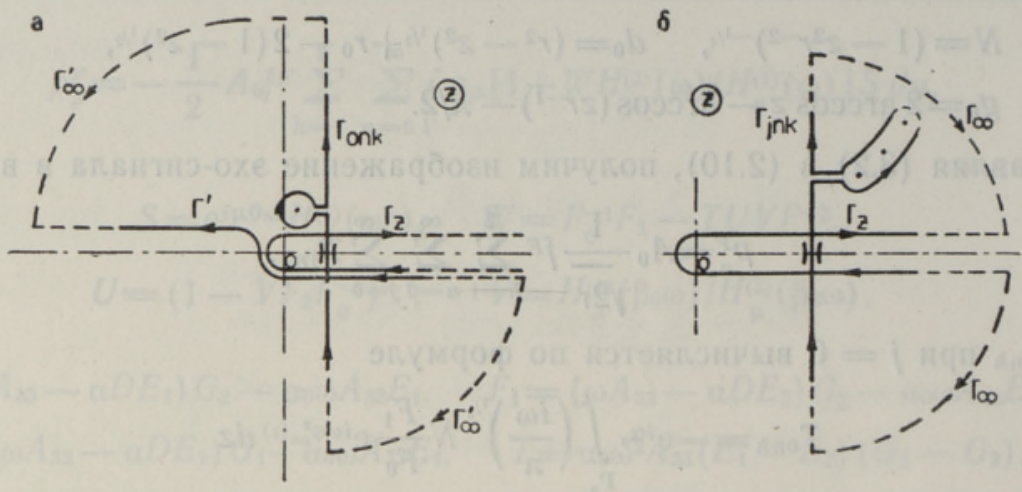

Рис. 1.

точки для (3.4) и справа от нее для (3.5). В случае отсутствия седловых точек расчет зависит от того, где не выполняется ограничение в соответствующем условии из (3.7) - (3.9): если сверху - учитываются вклады всех полюсов первой четверти плоскости $z$, если же снизу они в расчет не принимаются.

После вычисления вкладов седловых точек и полюсов, координаты $z=z_{s}$ которых определяются как решения уравнения $F_{0}=0$, интегралов (3.4) и (3.5), функции $T_{\text {jnk }}$ могут быть представлены в общем для любого значения $j$ в виде

$$
T_{j n k}=G_{j n k}^{0} \mathrm{e}^{i \omega d_{j}\left(z_{f n k}\right)}+\sum_{s=1}^{\infty} G_{j n k}^{s} \mathrm{e}^{i \omega q_{n k}^{\prime}\left(z_{s}\right)}, \quad j=0,1,2, \ldots
$$

Амплитудные функции $G_{j n k}^{0}$ и $G_{j n k}^{s}$ в (3.10) при $j=0$ вычисляются по формулам

$$
\begin{aligned}
G_{0 n k}^{0} & =\left[B C_{0} N F_{1} F_{0}^{-1}\right]_{z=z_{0 n k}}, \\
G_{0 n k}^{s} & =2 i \sqrt{i \pi \omega}\left[\frac{N F_{1}}{\partial F_{0} / \partial z}\right]_{z=z_{s}} H\left(\vartheta_{n k}-\vartheta_{s}\right)
\end{aligned}
$$

и при $j=1,2, \ldots-$ по формулам

$$
\begin{gathered}
G_{j n k}^{0}=\left[B C_{j} N T F_{2}^{j-1} F_{1}^{-j-1}\right]_{z=z_{n k}} \mathrm{e}^{i \alpha_{j},} \\
G_{j n k}^{s}=\frac{2 i \sqrt{i \pi \omega}}{\mathrm{e}^{i \omega \varphi_{n k}^{\prime}\left(z_{s}\right)}} \operatorname{Res}\left[\frac{T N F_{2}^{j-1}}{F_{0}^{j+1}} \mathrm{e}^{i \omega \varphi_{n k}^{\prime}(z)}\right]_{z=z_{s}} \mathrm{e}^{i \alpha_{j} H\left(\vartheta_{n k}-\vartheta_{s}\right) .}
\end{gathered}
$$

В формулах $(3.11)-(3.14)$ прй любом значении $j$

$$
\begin{gathered}
B=\left(1-z^{2}\right)^{1 / 4}, \quad \vartheta_{s}=-\operatorname{Re} g_{j}\left(z_{s}\right), \\
C_{j}=\left\{1-\frac{1}{2}\left(1-z^{2}\right)^{1 / 2}\left[\left(r^{2}-z^{2}\right)^{-1 / 2}+2 j \beta_{0}^{-1}\left(1-z^{2} \beta_{0}^{-2}\right)^{-1 / 2}\right]\right\}^{-1 / 2} .
\end{gathered}
$$

Если седловые точки отсутствуют, функции $G^{0}{ }_{j n k} \equiv 0$ при любом значении $j$. Функции $G_{j n k}^{s}$ в этом случае вычисляются по-прежнему по формулам (3.12) и (3.14); следует лишь брать $\vartheta_{s}=j \pi$. 
4. Анализ полученных результатов. Функции $T_{j n k}$ в (3.3) имеют смысл составляющих стационарного эхо, т. е. составляющих эхо, порожденных плоской стационарной зондирующей волной

$$
p_{i}=\sqrt{2 r} \mathrm{e}^{i \omega l} .
$$

Функции $G_{j n k}^{0}$ и $G_{j n k}^{s}$ в (3.10) определяют комплексные амплитуды, а функции $d_{j}$ и $\varphi_{n k}^{j}$ - времена прибытия отдельных составляющих стационарного эхо. Первый член в (3.10) - это составляющие, возбужденные волнами стационарного эхо, проходящими через оболочку в заполнитель, отражающимися $j-1$ раз от внутренней поверхности оболочки при распространении в заполнителе и вновь проходящими через оболочку в окружающую среду. При $j=0$ первый член в (3.10) явля. ется составляющей эхо, отраженной от внешней поверхности оболочки. Члены, находящиеся под знаком суммы по $s$ в (3.10), определяют составляющие, возбужденные волнами стационарного эхо, распространяющимися часть своего пути в оболочке и возле ее поверхности как периферические и ползущие, а часть пути как волны, проходящие через заполнитель. Во всех членах число $j$ показывает, сколько раз волна прошла через заполнитель. Члены $j=0$ определяют те составляющие эхо, которые имеют место как в случае пустой, так и в случае оболочки с жидким заполнителем, а члены $j=1,2, \ldots$ - те составляющие, которые имеют место только в случае заполненной жидкостью оболочки. Во всех членах число $n$ показывает, сколько полных оборотов вокруг оси волны совершили в оболочке или заполнителе до отправления к точке наблюдения. Число $k$ показывает, распространялись ли эти волны по часовой стрелке $(k=1)$ или против нее $(k=2)$. Число $s$ в $(3.10)$ указывает номер полюса, т. е. номер моды периферических или ползущих волн. В практических расчетах, как правило, достаточно учитывать только вклады безмоментной $(s=1)$ и изгибной $(s=2)$ мод периферических волн.
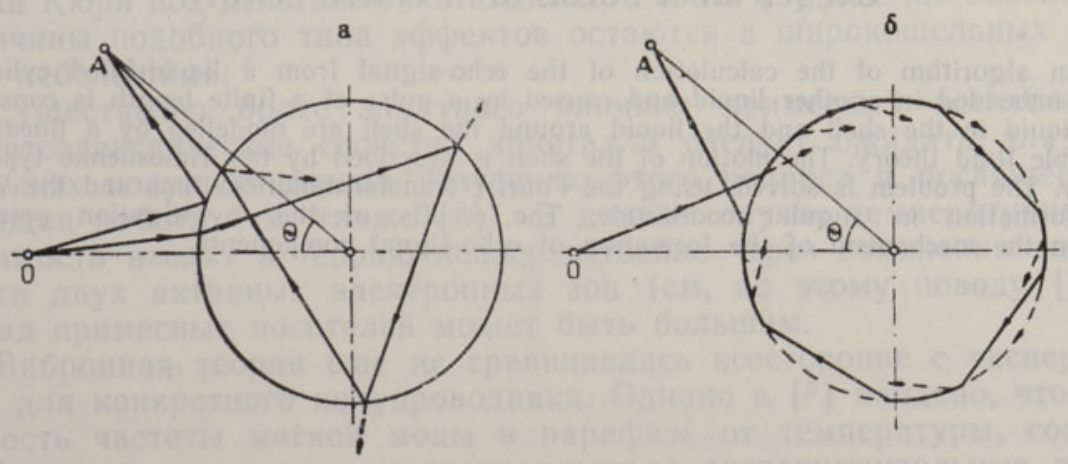

Рис. 2.

На рис. $2, a$ изображены пути от источника зондирующего импульса $O$ до точки наблюдения $A$ проходящих и отраженных от внутренней поверхности оболочки волн при $n=0, k=1, j=0$ и $n=1, k=1, j=3$. Рис. 2,б иллюстрирует один из бесконечно многих возможных путей одной моды периферических волн при $n=1, k=1, j=3$.

Преобразование Фурье по времени (2.1) обращается способом, предложенным в [6]. 


\section{ЛИТЕРАТ У Р А}

1. Junger M. C., J. Acoust. Soc. America, 24, No. 4, 366 (1952).

2. Л ямшев Л. М., Акуст. ж., 4, № 2, 161 (1958).

3. Белоозеров Н. Н., Долгова И. И., Акуст. ж., 16, № 3, 364 (1970).

4. Лиходаева Е. А., Шенде ров Е. Л., Акуст. ж., 17, № 1, 79 (1971).

5. Х ей фец Е. И., Ш енде ров Е. Л., Акуст. ж., 18, № 3, 456 (1972)

6. М е т с а в э эр Я. А., ПММ, 37, вып. 2, 274 (1973),

7. Brill D., Uberall H., J. Acoust. Soc. America, 47, No. 5, 1467 (1970).

8. B rill D., Uberall H., J. Acoust. Soc. America, 50, No. 3, 921 (1971).

9. K u m a r R., Acustica, 27, No. 6, 317 (1972).

10. Mirsky I., Herrman n G., J. Acoust. Soc. America, 29, No. 10, 1116 (1957).

Институт кибернетики

Академии наук Эстонской ССР
Поступила в редакцию 23/XII 1975

\section{J. METSAVEER, J. PIKK}

\section{VEDELIKUGA TÄIDETUD SILINDRILISELT KOORIKULT SAABUVA LOPLIKU IMPULSI KAJASIGNAAL}

Esitatakse vedelikuga täidetud silindriliselt koorikult saabuva lõpliku impulsi tekitatud kajasignaali arvutamise algoritm. Nii koorikus asuv kui ka teda ümbritsev vedelik on modelleeritud ideaalselt kokkusurutava vedeliku lineaarse teooria järgi. Kooriku liikumist on kirjeldatud Timošenko tüüpi lineaarse koorikute teooria kohaselt. Lahendi sōltuvus ajast leitakse Fourier' teisenduse ja sōltuvus nurgakoordinaadist Watsoni teisenduse abil. Saadud tulemuste põhjal kirjeldatakse kajasignaali erinevate komponentide moodustumise mehhanismi.

\section{J. METSAVEER, J. PIKK}

\section{THE ECHO-SIGNAL FROM A CYLINDRICAL SHELL FILLED WITH LIQUID, CAUSED BY A PULSE OF A FINITE LENGTH}

An algorithm of the calculation of the echo-signal from a liquid-filled cylindrical shell, imbedded in another liquid and caused by a pulse of a finite length is considered. The liquid in the shell and the liquid around the shell are modelled by a linear compressible fluid theory. The motion of the shell is described by the Timoshenko type shell theory. The problem is solved, using the Fourier transformation in time and the Watson transformation in angular coordinate. The results of the investigation permit to explain the mechanism of the formation of echo-signal components. 